\title{
Structural and optical properties of electron beam evaporated CdSe thin films
}

\author{
N J SUTHAN KISSINGER*, M JAYACHANDRAN", K PERUMAL ${ }^{\dagger}$ and \\ C SANJEEVI RAJA ${ }^{\dagger \dagger}$ \\ Nesamony Memorial Christian College, Marthandam 629 165, India \\ \#ECMS Division, Central Electrochemical Research Institute, Karaikudi 630 006, India \\ ${ }^{\dagger}$ SRMV College of Arts \& Science, Coimbatore 641 020, India \\ ${ }^{\dagger \dagger}$ Alagappa University, Karaikudi 630 003, India
}

MS received 5 July 2007; revised 14 August 2007

\begin{abstract}
Thin films of cadmium selenide (CdSe) as a semiconductor is well suited for opto-electronic applications such as photo detection or solar energy conversion, due to its optical and electrical properties, as well as its good chemical and mechanical stability. In order to explore the possibility of using this in optoelectronics, a preliminary and thorough study of optical and structural properties of the host material is an important step. Based on the above view, the structural and optical properties of CdSe films have been studied thoroughly in the present work. The host material, CdSe film, has been prepared by the physical vapour deposition method of electron beam evaporation (PVD: EBE) technique under a pressure of $5 \times 10^{-5} \mathrm{mbar}$. The structural properties have been studied by XRD technique. The hexagonal structure with a preferred orientation along the ( 00 2 $)$ direction of films has been confirmed by the X-ray diffraction analysis. The films have been analysed for optical band gap and absorbed a direct intrinsic band gap of $1.92 \mathrm{eV}$.
\end{abstract}

Keywords. CdSe films; electron beam evaporation; structural properties; energy band gap.

\section{Introduction}

$\mathrm{CdSe}$ is a direct band gap semiconductor belonging to the II-VI groups. Several physical and chemical techniques are available for the growth of CdSe thin films (Savadago and Mandal 1992; Murali et al 1993; Sarma et al 1993; Elango et al 2000). The structural and optical properties, as well as its good chemical and mechanical stability, recommends cadmium selenide ( $\mathrm{CdSe}$ ) as a semiconductor well suited for optoelectrical application, such as photodetection or solar energy conversion. It is a well known fact that the quality of the device based on CdSe thin films strongly depends on the structural and electronic properties of the films obtained by the various experimental conditions. The material has been grown in bulk single crystalline form and has been used as efficient photo detector. Cadmium selenide films have been grown by evaporation technique and used as gas sensors for the detection of oxygen (Smyntyna et al 1994). Cadmium selenide thin films have been grown by vacuum deposition technique (Mahmoud 1990).

The synthesis of binary metal chalcogenide of group II-VI semiconductors in a nanocrystalline form has been

*Author for correspondence (suthan.kissinger@gmail.com) a rapidly growing area of research due to its important non-linear optical properties, luminescent properties, quantum size effect and other important physical and chemical properties (Ghosh et al 2003). Semiconductor nanocrystallites belong to a state of matter in the transition region between molecules and solids. The physical and chemical properties of these nanocrystallites are found to be strongly size dependent. The material properties prepared by different methods are dependent on the nature of preparation and heat treatments like annealing in air, vacuum or different gaseous environments like $\mathrm{H}_{2}, \mathrm{~N}_{2}$, and Ar etc.

Cadmium selenide $(E g=1.7 \mathrm{eV})$ is one of the promising semiconducting materials that has been studied for application in solar cells (Richter 1988), $\gamma$-ray detectors (Roth 1989), thin film transistors (Van Calster et al 1988) etc. Freshly deposited thin films may contain many defects such as voids, pinholes etc. Annealing of thin films reduces the defects and increases the crystallite size along with recrystallization process. So many researchers have prepared $\mathrm{CdSe}$ thin films using various methods. However, very few have prepared CdSe thin films by electron beam evaporation technique. Kainthla et al (1980) reported the optical and structural properties of chemically deposited CdSe thin films onto various substrates (glass, mica, Si and Ge). Kale and Lokhande (2004) reported that as-deposited $\mathrm{CdSe}$ films were red in colour and 
showed quantum-size effect when deposited at lower temperatures. Several workers (Fortin et al 1985; De and Mishra 1997) studied the oxygen absorption and desorption energies in CdSe thin films. Samanta et al (1996) studied the photoconductivity of the as-grown thermally evaporated CdSe thin films.

It is important that the improvement of materials properties requires a closer inspection of preparation conditions. From this point of view, a large number of techniques for preparing CdSe films were employed out of which the electron beam evaporation technique, one of the physical vapour deposition methods, has been widely used for the growth of device quality thin films (Miyake et al 1984). In the present paper, we have investigated the structural, morphological and optical properties of electron beam evaporated CdSe films.

\section{Experimental}

Substrate cleaning plays an important role in the deposition of thin films. Commercially available glass micro slides were boiled in chromic acid for $2 \mathrm{~h}$, washed with detergent, rinsed in acetone and finally they were ultrasonically cleaned with double distilled water before use.

Thin films of CdSe were deposited from $\mathrm{CdSe}$ powder (Aldrich, 99.99\%) by electron beam evaporation technique using a HINDHIVAC Vacuum coating unit (model: 12A4D) fitted with electron beam power supply (model: EBG-PS-3K). The CdSe films were prepared at different thicknesses on well degreased microscopic glass plates for the present study. The dry $\mathrm{CdSe}$ powder was made into pellets, taken in a graphite crucible. The pelletized CdSe targets were heated by means of an electron beam collimated from the d.c. heated tungsten filament cathode. The surface of the CdSe pellet was bombarded by $180^{\circ}$ deflected electron beam with an accelerating voltage of $6 \mathrm{kV}$ and a power density of about $1.5 \mathrm{~kW} / \mathrm{cm}^{2}$. The evaporated particles from $\mathrm{CdSe}$ pellet were deposited as thin films on glass substrates at a pressure of about $5 \times 10^{-5}$ mbar. Each substrate was placed normal to the line of sight from the evaporation source at a different polar angle to obtain uniform deposition. CdSe films were deposited on glass substrates for four different thicknesses at room temperature (RT). The different preparation parameters such as source to substrate distance $(12 \mathrm{~cm})$ and partial pressure $\left(10^{-5} \mathrm{mbar}\right)$ were varied and optimized for depositing uniform, well adherent and transparent films. The films were found to be uniform, porous free and adhered well with the glass plates. The thickness of the film was measured by stylus method.

The structural properties of the films were studied by the JEOL JDX X-ray diffractometer (XRD) using $\mathrm{CuK} \alpha$ radiation $(\lambda=1.5418 \AA)$ with $\mathrm{Ni}$ filter. The optical spectra were recorded in the wavelength range $500-2500 \mathrm{~nm}$ using UV-Vis-NIR spectrophotometer (Hitachi V-3400).
HITACHI S-30000H scanning electron microscope has been used for the surface morphological analysis of electron beam evaporated $\mathrm{CdSe}$ thin films prepared at room temperature.

\section{Results and discussion}

\subsection{X-ray diffraction studies}

Cadmium selenide can form the hexagonal, wurtzite-type structure or the cubic, zinc blende-type structure (JCPDS files No. 8-459 and 19-191). The X-ray diffraction patterns of electron beam evaporated $\mathrm{CdSe}$ films on glass substrates at room temperature $\left(T_{\text {sub }}=\mathrm{RT}\right)$, having thicknesses 710, 290, 160, $120 \mathrm{~nm}$ are shown in figures 2, 3, 4 and 5, respectively. Figure 1 shows the X-ray diffractogram (XRD) of CdSe powder which was used for deposition. The observation of X-ray peaks in all the four CdSe films shows that the electron beam evaporated films are polycrystalline in nature. Table 2 lists the observed $d$ values with the standard JCPDS $d$-values for CdSe thin films. The observed $d$-values are in good agreement with the standard values for the hexagonal structure. The XRD patterns for these four electron beam evaporated $\mathrm{CdSe}$ thin films of different thicknesses indicate $\left(\begin{array}{lll}0 & 0 & 2\end{array}\right)$ reflection. It shows that the preferred orientation lies along $\left(\begin{array}{lll}0 & 0 & 2\end{array}\right)$ direction in electron beam evaporated $\mathrm{CdSe}$ thin films.

The strong X-ray peak at $\left(\begin{array}{lll}0 & 0 & 2\end{array}\right)$ plane indicates the preferred orientation of the crystalline grains with $c$-axis perpendicular to the plane of the substrate that makes the (lll $\left.\begin{array}{ll}0 & 0\end{array}\right)$ peak relatively stronger than the other peaks. The crystalline size (grain diameter $(D)$ ), of the crystallites can be determined using the Scherrer's formula from the full width at half maximum (FWHM) $\beta$ (Wilson 1963),

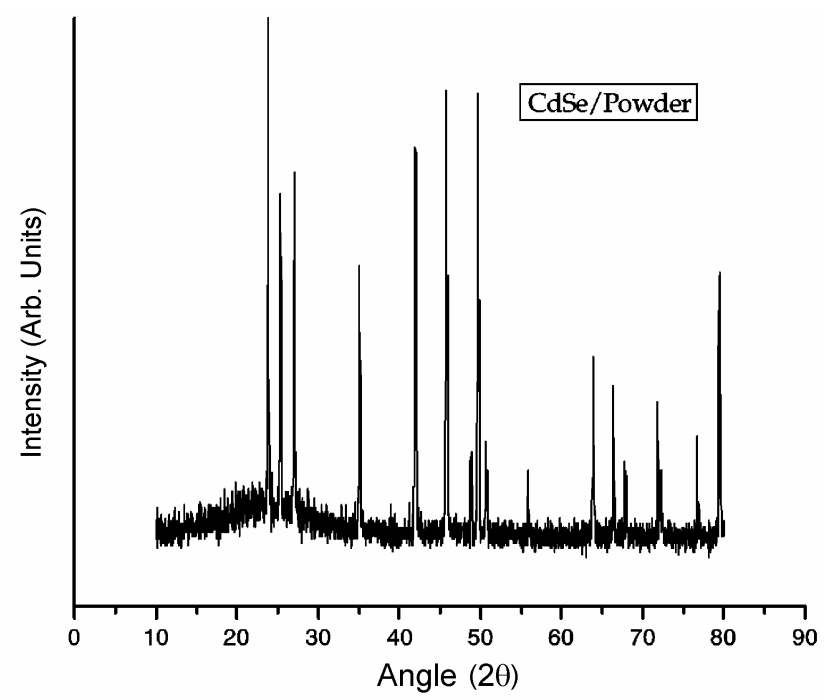

Figure 1. X-ray diffractogram of bulk CdSe. 
Table 1. Structural parameters of CdSe films deposited for various thicknesses.

\begin{tabular}{lccc}
\hline Thickness $(\mathrm{nm})$ & Grain size, $D(\mathrm{~nm})$ & Strain $(\varepsilon) \times 10^{-4}\left(\operatorname{lin}^{-2} \mathrm{~m}^{-4}\right)$ & $\begin{array}{c}\text { Dislocation density } \\
(\delta) \times 10^{14}\left(\operatorname{lin~}^{-2}\right)\end{array}$ \\
\hline 120 & 24 & $16 \cdot 61$ & 14.75 \\
160 & 31 & $9 \cdot 88$ & 11.38 \\
290 & 42 & $5 \cdot 56$ & 8.53 \\
710 & 46 & $4 \cdot 60$ & 7.77 \\
Bulk & 64 & $3 \cdot 31$ & 5.35 \\
\hline
\end{tabular}

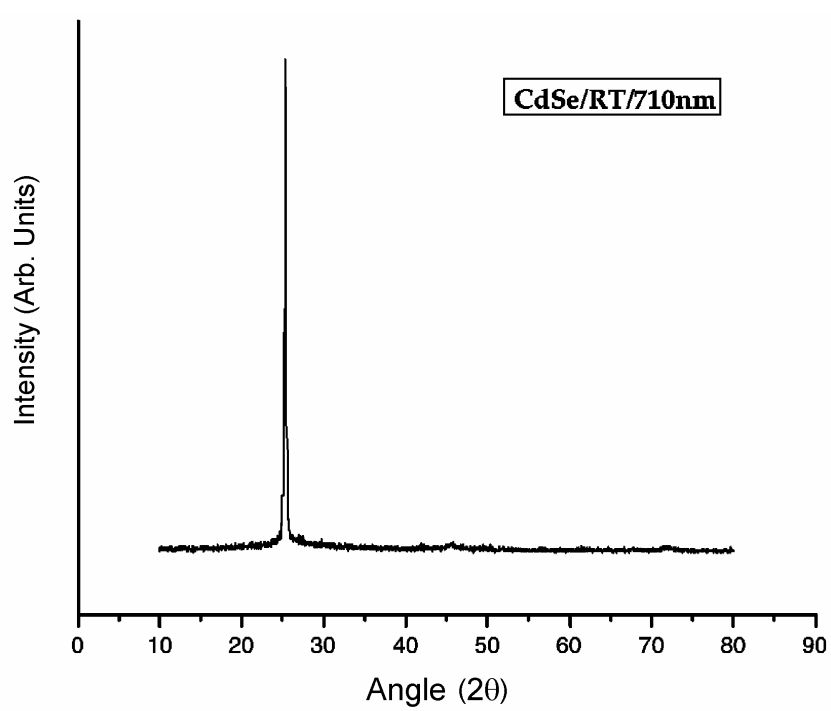

Figure 2. X-ray diffractogram of CdSe film of thickness, $710 \mathrm{~nm}$.

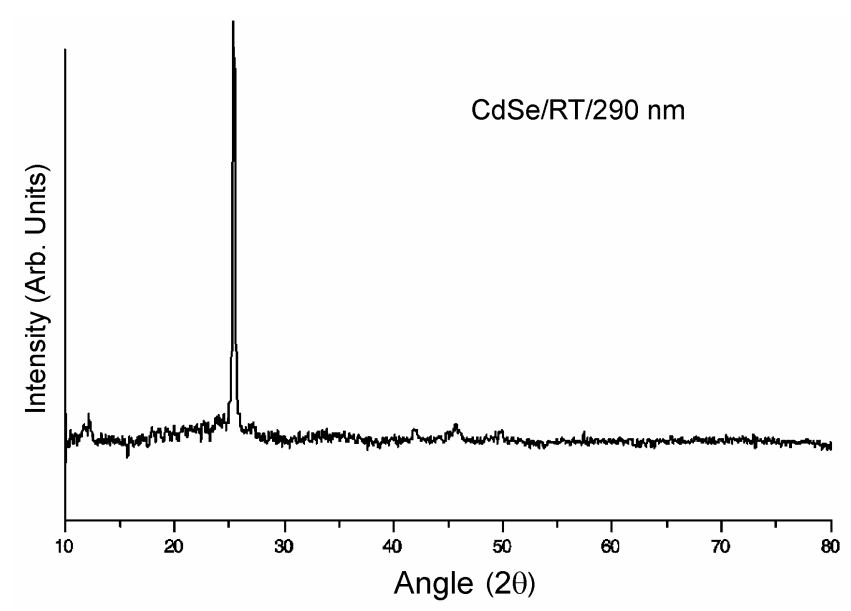

Figure 3. X-ray diffractogram of thickness, $290 \mathrm{~nm}$.

$$
D=0.94 \lambda / \beta \cos \theta,
$$

where $\lambda$ is the wavelength of X-ray used, $\beta$ the FWHM, $D$ the particle size and $\theta$ the angle between the incident and scattered X-ray. The strain values, $\varepsilon$, can be evaluated by using the following relation

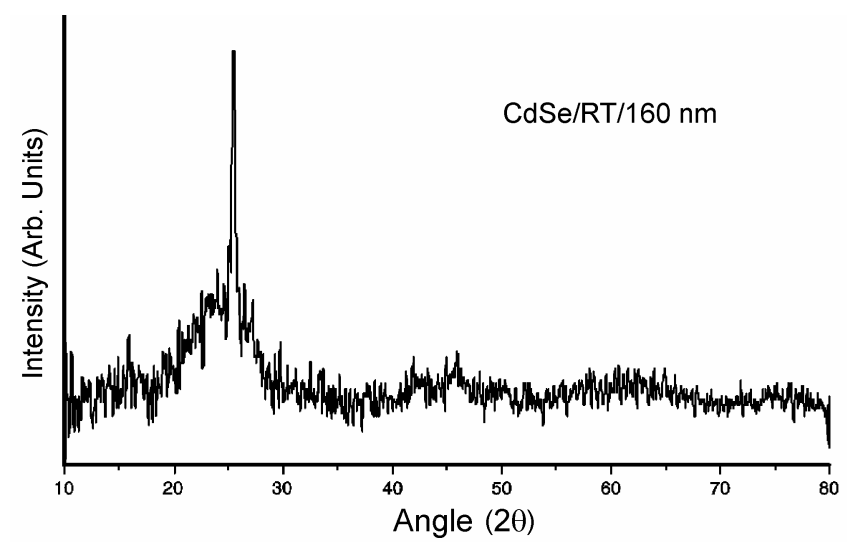

Figure 4. X-ray diffractogram of CdSe film of thickness, $160 \mathrm{~nm}$.

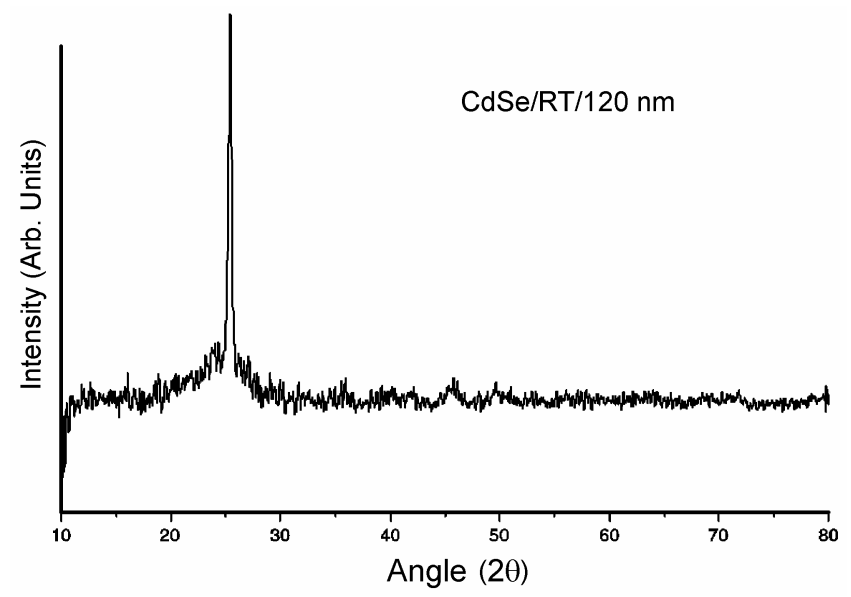

Figure 5. X-ray diffractogram of thickness, $120 \mathrm{~nm}$.

$$
\varepsilon=\beta \cos \theta / 4
$$

The lattice spacing, $d$, is calculated from the Bragg's formula

$$
d=\lambda / 2 \sin \theta
$$

The lattice parameter, $c$, is determined for hexagonal structure by the following expression

$$
1 / d^{2}=\left(h^{2}+k^{2}+l^{2}\right) / c^{2},
$$

where $h, k$ and $l$ represent the lattice planes. The dislocation density, $\delta$, has been calculated by using the formula (De and Mishra 1997), 
Table 2. Structural parameters of CdSe films deposited for various thicknesses.

\begin{tabular}{lcccccc}
\hline & & & \multicolumn{2}{c}{ Lattice spacing, $d(\AA)$} & \\
\cline { 5 - 6 } Thickness $(\mathrm{nm})$ & $2 \theta($ deg.) & $h k l$ & Experimental & Standard & Lattice \\
parameter, $c(\AA)$
\end{tabular}

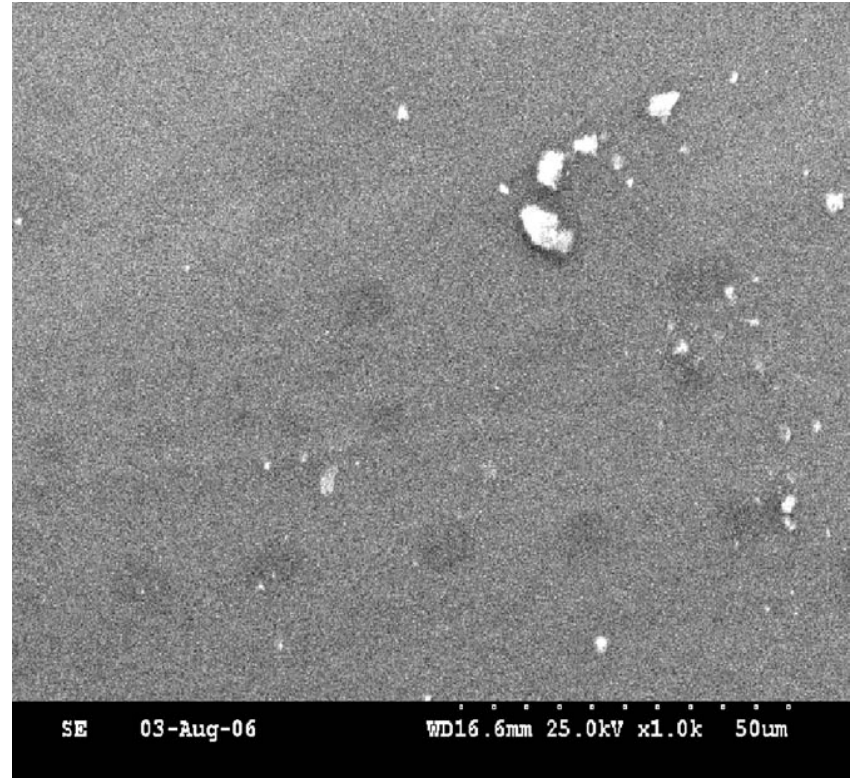

Figure 6. SEM picture of CdSe.

$$
\delta=15 \varepsilon / a D .
$$

The crystallite size and dislocation density of the electron beam evaporated $\mathrm{CdSe}$ thin films are calculated and given in table 1 . The intense and sharp peaks in X-ray diffraction pattern reveal the good crystallinity of the films and also confirm the stoichiometric nature of $\mathrm{CdSe}$ films.

In the present case, XRD patterns of the films deposited for different thicknesses were studied. The films of $\mathrm{CdSe}$ prepared by the present method are nanocrystalline in nature and have hexagonal crystal structure. From the full width at half maximum, we have calculated the grain size of CdSe powder as well as films. Grain size of CdSe films was estimated using Scherrer's formula (1).

All the peaks corresponding to (100), (002), (101), (110), (103) and (112) reflections are observed. These reflections indicate the formation of single phase material.

The peaks corresponding to hexagonal CdSe are observed. Elemental Se (or) Cd peak was not observed. The intensity of the peak corresponding to (002) orientation is observed in all cases. The peak corresponding to (002) plane indicates the preferential orientation in this direction.
Table 1 shows the variation of grain size with thickness. It was found that the thickness of the film was in the range $100-750 \mathrm{~nm}$. The grain sizes of CdSe films were varied by depositing films at different thicknesses. As the thickness decreased from $710-120 \mathrm{~nm}$, the grain size also decreased from $46-24 \mathrm{~nm}$. The as deposited films were uniform and adhered well to the substrates.

The lattice constant, ' $c$ ', calculated using the XRD data for different $\mathrm{CdSe}$ films are given in table 2, which are in close agreement with the ASTM data. Table 2 gives the comparison of observed ' $d$ ' values with the standard JCPDS data files. The most dominant plane $\left(\begin{array}{lll}0 & 0 & 2\end{array}\right)$ was observed in all the films. The film with thickness, $290 \mathrm{~nm}$, gives the lattice constant value as $7.014 \AA$ which is approximately equal to the standard value. The strain and dislocation density of electron beam evaporated $\mathrm{CdSe}$ films for various thicknesses are tabulated in table 2 . The strain $(\varepsilon)$ and dislocation density $(\delta)$ of the films varies from $16.6 \mathrm{lin}^{-2} \mathrm{~m}^{-4}$ to $4.6 \mathrm{lin}^{-2} \mathrm{~m}^{-4}$ and $14.7 \mathrm{lin} \mathrm{m}^{-2}$ to 7.7 lin $\mathrm{m}^{-2}$ as the thickness of the film increases from $120-710 \mathrm{~nm}$. For the bulk CdSe the strain and dislocation

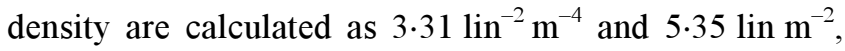
respectively.

\subsection{Scanning electron microscopic studies}

Scanning electron microscopy (SEM) is a convenient technique to study microstructure of thin films. HITACHI S$30000 \mathrm{H}$ scanning electron microscope has been used for the surface morphological analysis of electron beam evaporated $\mathrm{CdSe}$ thin films prepared at room temperature. The SEM picture of as-deposited ( $\left.T_{\text {sub }}=\mathrm{RT}\right) \mathrm{CdSe}$ films on glass substrate is shown in figure 6. It is clearly observed from the surface morphological studies by SEM that the as-deposited CdSe films are nanocrystalline, homogenous, without cracks or holes and well covered to the glass substrate.

\subsection{Optical absorption studies by UV-Vis-NIR spectrophotometer}

The optical absorption and transmittance measurements of $\mathrm{CdSe}$ thin films have been carried out at room temperature by placing an uncoated identical conducting glass 


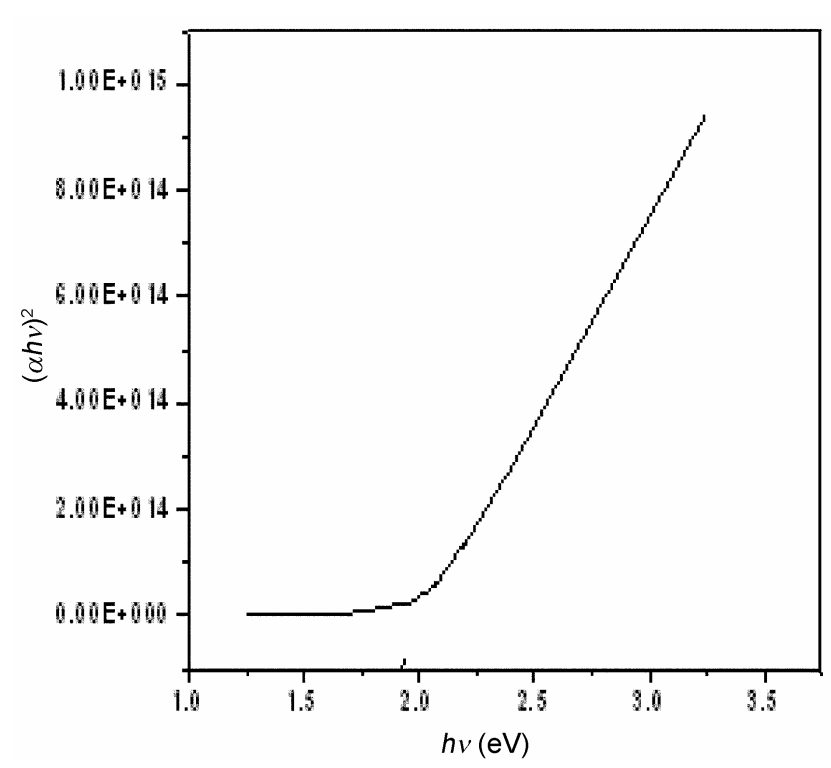

Figure 7. Band gap of CdSe at RT.

substrate in the reference beam. The optical spectra of electron beam evaporated CdSe thin films have been recorded in the wavelength range $500-2500 \mathrm{~nm}$. A plot of $(\alpha h v)^{2}$ vs $h v$ of CdSe thin films for three different thicknesses prepared at room temperature (RT) is shown in figure 7 .

From the graph, the direct band gap energies are evaluated. Extrapolating the straight-line portions of the plot $(\alpha h v)^{2}$ vs $h v$ for zero absorption coefficient value give the energy band gap value of ' $E g$ '. The band gap values for the films prepared at room temperature $\left(T_{\text {sub }}=\mathrm{RT}\right)$ is calculated. It is found that the band gap value is $1.92 \mathrm{eV}$ for the as-deposited CdSe thin films.

\section{Conclusions}

The present study indicates that the electron beam evaporation technique can be successfully employed for the preparation of quality $\mathrm{CdSe}$ thin films. The deposited films grow in nanocrystalline phase with $\left(\begin{array}{lll}0 & 0 & 2\end{array}\right)$ hexagonal plane orientation. The crystalline size of the particles increases as a result of increasing film thickness. Further the strain $(\varepsilon)$ and dislocation density $(\delta)$ decreased as the grain size increased. The quantum efficiency may be enhanced further by optimizing the deposition conditions and film thickness.

\section{References}

De C K and Mishra N K 1997 Indian J. Phys. 71530

Elango T, Subramanian V and Murali K R 2000 Surf. Coat. Technol. 1238

Fortin B, Larzul H, Lebigot J, Raoult F and Rosse G 1985 Electron. Opt. 13151

Ghosh S, Mukherjee A, Kim H and Lie C 2003 Mater. Chem. Phys. $\mathbf{7 8} 726$

Hodes G 1980 Nature 28529

JCPDS files No. 08-459 and 19-191

Kainthla R C, Pandya D K and Chopra K L 1980 J. Electrochem. Soc. 127277

Kale R B and Lokhande C D 2004 Appl. Surf. Sci. 223343

Mahmoud A H 1990 Cryst. Res. Technol. 251147

Miyake K, Kaneko H, Sano M and Suedomi N 1984 J. Appl. Phys. 552747

Murali K R, Subramanian V, Rangarajan N, Lakshmanan A S and Rangarajan S K 1993 Bull. Electrochem. 9209

Richter H 1988 20th IEEE photovoltaics specialists conf. (New York: IEEE) p. 1537

Roth M 1989 Nucl. Instrum. Methods A283 291

Samanta D, Samanta B, Chaudhuri A K, Ghorai S and Pal U 1996 Semicond. Sci. Technol. 11548

Sarma H P, Rangarajan N and Murali K R 1993 Phys. Status Solidi A148 209

Savadago O and Mandal K C 1992 Mater. Chem. Phys. 31301

Smyntyna V A, Gersutenko V, Sashulis S, Mattogno G and Reghini S 1994 Sensors \& Actuators B18 464

Van Calster A, Vervaet A, DeRycke I, De Baets J and Vanfleteren J 1988 J. Cryst. Growth 86924

Wilson A P J 1963 Mathematical theory of X-ray powder diffractometry (New York: Gordon and Breach) p. 62 